\title{
Relationship between Kita-Kyushu Lung Cancer antigen-1 expression and prognosis of cases with lung squamous cell carcinoma
}

\author{
Yoshinobu Ichiki ${ }^{1,2}$, Takashi Fukuyama ${ }^{3}$, Haruki Ohmiya ${ }^{3}$, Mari Ueno ${ }^{4}$, Shinya Yanagi ${ }^{4}$, Yoshiro Kanasaki ${ }^{1}$, \\ Hidenori Goto ${ }^{1}$, Shuji Mikami ${ }^{4}$, Hitoshi Yamazaki ${ }^{5}$, Kozo Nakanishi ${ }^{1}$, Tsuyoshi Ishida ${ }^{4}$ \\ ${ }^{1}$ Department of General Thoracic Surgery, National Hospital Organization, Saitama Hospital, Wako, Japan; ${ }^{2}$ Second Department of Surgery, \\ University of Occupational and Environmental Health, School of Medicine, Kitakyushu, Japan; ${ }^{3}$ Division of Biomedical Research, Kitasato \\ University Medical Center, Kitamoto, Japan; ${ }^{4}$ Department of Diagnostic Pathology, National Hospital Organization, Saitama Hospital, Wako, Japan; \\ ${ }^{5}$ Division of Pathology, Kitasato University Medical Center, Kitamoto, Japan \\ Contributions: (I) Conception and design: Y Ichiki; (II) Administrative support: Y Ichiki, T Fukuyama; (III) Provision of study materials or patients: \\ Y Ichiki; (IV) Collection and assembly of data: Y Ichiki, S Yanagi, Y Kanasaki, H Goto, K Nakanishi; (V) Data analysis and interpretation: Y Ichiki, \\ T Fukuyama, H Omiya, M Ueno, S Mikami, H Yamazaki, T Ishida; (VI) Manuscript writing: All authors; (VII) Final approval of manuscript: All \\ authors. \\ Correspondence to: Yoshinobu Ichiki, MD, PhD. Department of General Thoracic Surgery, National Hospital Organization Saitama Hospital, 2-1 \\ Suwa, Wako, Saitama, 351-0102, Japan. Email: y-ichiki@med.uoeh-u.ac.jp.
}

Background: Previously, we identified the highly immunogenic cancer testicular antigen named KitaKyushu Lung Cancer antigen-1 (KK-LC-1). In this study, we analyzed the effect of KK-LC-1 expression on the prognosis of patients with resected squamous cell lung cancer.

Methods: Fifty squamous cell lung cancer patients, who received complete resection, were enrolled in this study. The expressions of KK-LC-1, CD8, human leukocyte antigen (HLA) class I, and programmed cell death protein ligand-1 (PD-L1) were assessed via immunohistochemistry staining using the specimens obtained from the participants. The association between the expression of the abovementioned molecules and patient prognosis was investigated.

Results: KK-LC-1 expression was observed in 21 of 50 recruited cases (42\%). However, no significant correlation was found between KK-LC-1 expression and patient prognosis. The prognosis was significantly better in lung cancer cases with KK-LC-1 expression in which $\mathrm{CD}^{+} \mathrm{T}$ cells infiltrated the tumor. Regardless of the HLA class I expression or the PD-L1 expression, the KK-LC-1 expression in squamous cell lung cancer could not be detected as a significant prognostic factor. Furthermore, considering the polarity of the cancer tissue as epithelium, staining of KK-LC-1 tended to be strong in the area corresponding to the basal side of the tumor tissue. The Ki-67 expression was frequently observed in cancer cells on the basal side, which was consistent with the KK-LC-1 expression in representative four cases with KK-LC-1-positive squamous cell lung cancer.

Conclusions: Our results indicated that lung squamous cell cancer patients with KK-LC-1 expression and the tumor infiltrating $\mathrm{CD} 8^{+} \mathrm{T}$ cells might exhibit better prognosis. KK-LC-1 might be highly expressed in cancer cells with high proliferative capacity. Larger cohort analysis is still required for further elucidation and validation of the results of this study.

Keywords: Lung cancer; Kita-Kyushu Lung Cancer antigen-1 (KK-LC-1); cytotoxic T lymphocyte (CTL); programmed cell death protein ligand-1 (PD-L1); tumor immunology

Submitted Aug 09, 2021. Accepted for publication Nov 04, 2021.

doi: $10.21037 /$ tcr-21-1581

View this article at: https://dx.doi.org/10.21037/tcr-21-1581 


\section{Introduction}

Previously, we identified the cancer testis antigen KitaKyushu Lung Cancer antigen-1 (KK-LC-1) via cDNA expression cloning method using tumor-specific cytotoxic T lymphocyte (CTL) clone (1). KK-LC-1 is mapped to chromosome $\mathrm{Xq} 22$ and is not expressed in normal tissues except the testis; $33 \%$ of non-small cell lung cancers expressed it (1). In addition, we isolated $\mathrm{T}$ cell receptor (TCR) from KK-LC-1-specific CTL clones and transferred them into gd T cells. KK-LC-1-specific TCR-introduced gd T cells exhibited KK-LC-1-specific activity both in vivo and in vitro (2). A dominant KK-LC-1-specific immune response was previously observed in human papillomaviruspositive cervical cancer patients who completely regressed after adoptive immunotherapy (3). KK-LC-1 is a cancer testis antigen that is specifically expressed in cancer cells and has high immunogenicity, but its application to immunotherapy is still awaited.

Immune checkpoint inhibitors (ICIs) have made it possible to prolong the survival of lung cancer patients and have become a standard treatment for lung cancer. However, the response rate of ICI monotherapy is about $20 \%$ to $40 \%$ (4-8), and it is considered, to this end, that there are many aspects that still need to be improved. Applying the KK-LC-1-specific immune response to ICI might lead to better results.

Herein, the significance of the KK-LC-1 expression on the immunological microenvironment and prognosis of lung squamous cell cancer patients were analyzed. We present the following article in accordance with REMARK reporting checklist (available at https://dx.doi.org/10.21037/ tcr-21-1581).

\section{Methods}

\section{Patients and samples}

From August 2009 to June 2019, we analyzed 50 lung squamous cell cancer patients who received complete resection at our hospital. The pathological stage (p-stage) was determined according to the latest tumor, lymph node, and metastasis (TNM) classification (9). The relationship between these clinicopathologic factors and the KK-LC-1 expression in cancer cells was retrospectively analyzed.

This study was conducted in accordance with the Declaration of Helsinki (as revised in 2013). The study was approved by Saitama Hospital Ethics Committee (R201901 ) and informed consent was taken from all the patients.

\section{Immunobistochemistry (IHC)}

The Benchmark XT autostainer (Ventana Medical System, Tucson, AZ, USA) with anti-CD8 (SP57), anti-programmed cell death protein ligand-1 (anti-PD-L1) (SP263), antihuman leukocyte antigen (anti-HLA) class I HLA-A, B, C (D370-3H) (EMR8-5.1) MBL and anti-Ki-67 (309) was used for immunohistochemistry analysis (IHC) by using serial sections from paraffin-embedded tumors. Two pathologists counted the proportion of tumor stromal areas where IHC-positive lymphocytes existed to evaluate tumor infiltration of $\mathrm{CD}^{+}$lymphocytes. The median of the obtained values was applied for the analysis. We counted the proportion of cancer cells expressing the HLA class I among the viable cancer cells to assess the expression of HLA class I. To evaluate the PD-L1 expression in tumor stromal area, the proportion of IHC-positive immune cells among the immune cells in tumor stromal area was calculated. The proportion of IHC-positive immune cells in the immune cells in the tumor stromal area was counted to assess the PD-L1 expression in tumor infiltrating immune cells. The ratio of the area of IHC-positive cancer cells to the total area of viable cancer cells was then calculated to evaluate the PD-L1 expression in cancer cells, as we previously reported (10). The KK-LC-1 protein expression was evaluated in lung cancer tissue using the original monoclonal antibody against KK-LC-1 (Kmab34B3), which was constructed in collaboration with Dr. Fukuyama and CLEA Japan, Inc. (Tokyo, Japan). Cytoplasm and/or nucleus in cancer cells appearing as brown granules were defined as immunoreactivities for positive KK-LC-1 expression.

\section{Statistical analyses}

Correlation between KK-LC-1 and clinicopathological factors was analyzed using Fisher's Exact test. The KaplanMeier method was used for the survival analysis, the logrank test was used for the univariate analysis, and a Cox proportional hazard model was used for the multivariate analysis. The survival analysis, univariate analysis, and multivariate analysis were calculated using the KaplanMeier method, log-rank test, and Cox proportional hazard model, respectively.

A receiver operating characteristic (ROC) curve analysis was performed using the obtained clinicopathological data, and the cut-off values was calculated. All $\mathrm{P}$ values were twosided. Statistical significance was defined by $\mathrm{P}$ value was $<0.05$. The statistical analysis software used in this study was 
Table 1 The patients' characteristics are shown

\begin{tabular}{|c|c|c|c|}
\hline \multirow{2}{*}{ Factor } & \multicolumn{2}{|c|}{ KK-LC-1 } & \multirow{2}{*}{ P-value } \\
\hline & Positive & Negative & \\
\hline \multicolumn{4}{|c|}{ Age (years), n (\%) } \\
\hline$<70$ & $6(46.2)$ & $7(53.8)$ & 0.75 \\
\hline$\geq 70$ & $15(40.5)$ & $22(59.5)$ & \\
\hline \multicolumn{4}{|l|}{ Sex, n (\%) } \\
\hline Male & $14(37.8)$ & $23(62.2)$ & 0.35 \\
\hline Female & $7(53.8)$ & $6(46.2)$ & \\
\hline \multicolumn{4}{|c|}{ Brinkman index, median (range) } \\
\hline$<1,000$ & $7(29.2)$ & $17(70.8)$ & 0.09 \\
\hline$\geq 1,000$ & $14(53.8)$ & $12(46.2)$ & \\
\hline \multicolumn{4}{|c|}{ Performance status, n (\%) } \\
\hline 0 & $19(45.2)$ & $23(54.8)$ & 0.44 \\
\hline 1 & $2(25.0)$ & $6(75.0)$ & \\
\hline \multicolumn{4}{|l|}{$\mathrm{p}-\mathrm{T}, \mathrm{n}(\%)$} \\
\hline $1-2$ & $18(41.9)$ & $25(58.1)$ & 1.00 \\
\hline $3-4$ & $3(42.9)$ & $4(57.1)$ & \\
\hline \multicolumn{4}{|c|}{$\mathrm{p}-\mathrm{N}, \mathrm{n}(\%)$} \\
\hline $0-1$ & $21(42.9)$ & $28(57.1)$ & 1.00 \\
\hline 2 & $0(0)$ & $1(100)$ & \\
\hline
\end{tabular}

KK-LC-1, Kita-Kyushu Lung Cancer antigen-1.

EZR (Saitama Medical Center, Jichi Medical University, Saitama, Japan), which is a graphical user interface of $\mathrm{R}$ (The R Foundation for Statistical Computing, Vienna, Austria). EZR is a modified version of the $\mathrm{R}$ commander with the addition of statistical functions frequently used in biostatistics (11).

\section{Results}

\section{Patient characteristics}

Table 1 shows the patients' characteristics. Thirty-seven men $(74 \%)$ and 13 women (26\%) were included, with a median age at surgery of 74 years (range, 57-88 years). The numbers of cases with Eastern Cooperative Oncology Group (ECOG) performance status (PS) 0 and 1 cases were $42(84 \%)$ and $8(16 \%)$, respectively. The numbers of cases with p-stages I, II, and III were 36, 11, and 3, respectively. KK-LC-1 expression was observed in 21 of 50 recruited cases $(42 \%)$. There were no significant differences in clinicopathological factors between KK-LC-1-positive and -negative cases. The observation period was 16-3,643 days (median: 1,226 days). The median observation period was 1,226 days (16-3,643 days). The 5-year cancer-specific survival rate and disease-free survival rate were $76.7 \%$ and $71.5 \%$, respectively. None of the survivors reached the median survival period (Figure 1).

\section{Pathological analyses}

Figure 2 shows a statistical analysis using the data derived from IHC. The sensitivity and specificity were calculated from the ROC curve, and the maximum point was used as the cut-off value. Representative positive and negative cases of the PD-L1 and HLA class I expression of cancer cells (Figure 3), and the CD8 and PD-L1 expression of tumor infiltrating immune cells (Figure 4) by using an immunohistochemistry staining are shown.

\section{Cancer-specific survival}

As shown in the Figure 3, the specimens that exhibited staining in the cytoplasm and nucleus of lung cancer cells were evaluated as positive (Figure $5 A$ ), and those without staining were evaluated as negative (Figure $5 B$ ). Differences in prognosis were evaluated on the basis of KK-LC-1 expression status of lung cancer cells, but no significant difference was observed (Figure $5 C$ ). As shown in Figure $6 A, 6 B$, the prognosis was significantly better in cases in which lung cancer cells expressed KK-LC- 1 and CD8 ${ }^{+} \mathrm{T}$ cells infiltrated into the tumor. On the other hand, even if the lung cancer cells that expressed KK-LC-1 also expressed HLA-class I or PD-L1, it did not affect patient prognosis (Figure 6C-6E).

\section{Distribution of the KK-LC-1 expression}

During the KK-LC-1 expression analysis, we observed strong staining in the region corresponding to the basal side of the tumor tissue. Therefore, the relationship between the proliferative ability of cancer cells and the KK-LC-1 expression in cancer cells was confirmed via IHC staining for the Ki-67 expression in four representative cases with KK-LC-1-positive lung squamous cell carcinoma. The Ki67 expression in cancer cells was frequently observed on the basal side, which was consistent with the KK-LC-1 expression in all four cases (Figure 7). 

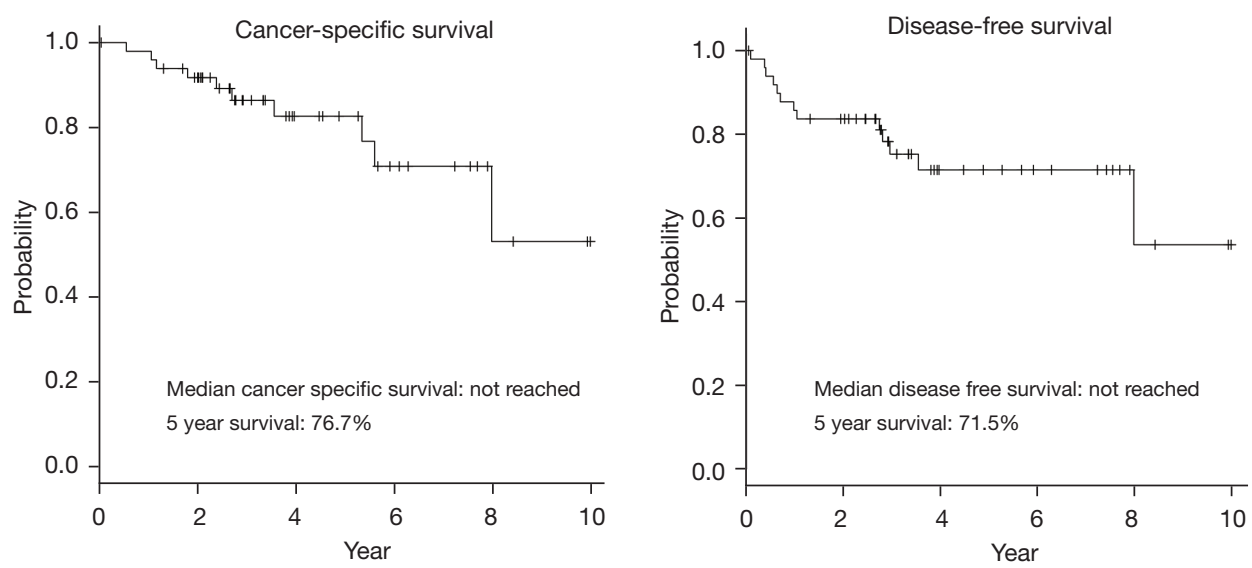

Figure 1 Kaplan-Meier curves for the cancer-specific and disease-free survival of lung squamous cell carcinoma patients are shown. The 5 -year cancer-specific survival rate and disease-free survival rate were $76.7 \%$ and $71.5 \%$, respectively.
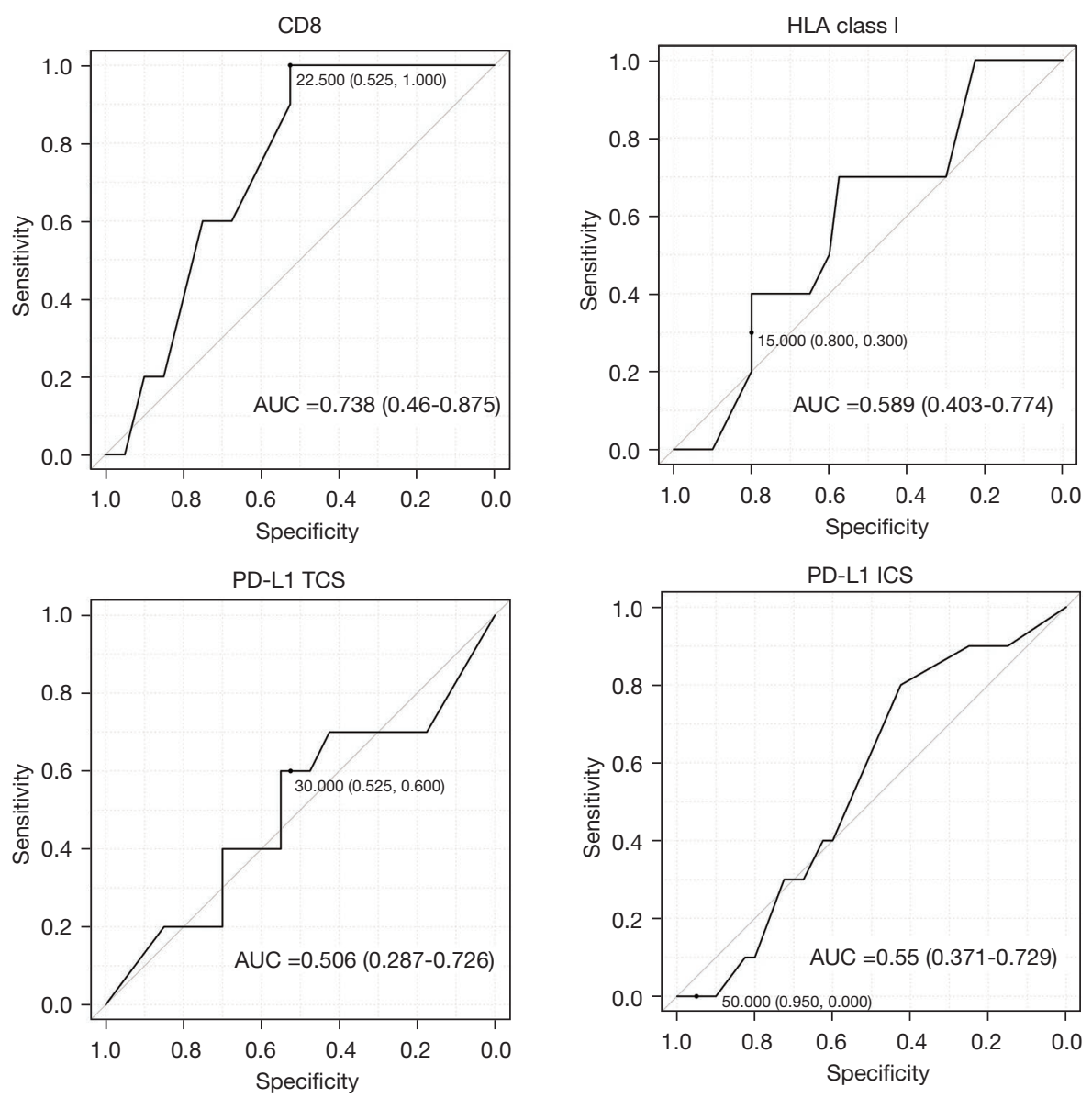

Figure 2 A ROC curve using which the sensitivity and the specificity to the cancer-specific survival were calculated, and the cut-off was determined. The cut-off values for CD8, HLA class I, PD-L1 TCS, and PD-L1 ICS were 22.5, 15.0, 30.0, and 50.0, respectively. ROC, receiver operating characteristics. HLA, human leukocyte antigen; PD-L1, programmed cell death protein ligand-1; TCS, tumor cells; ICS, immune cells. 

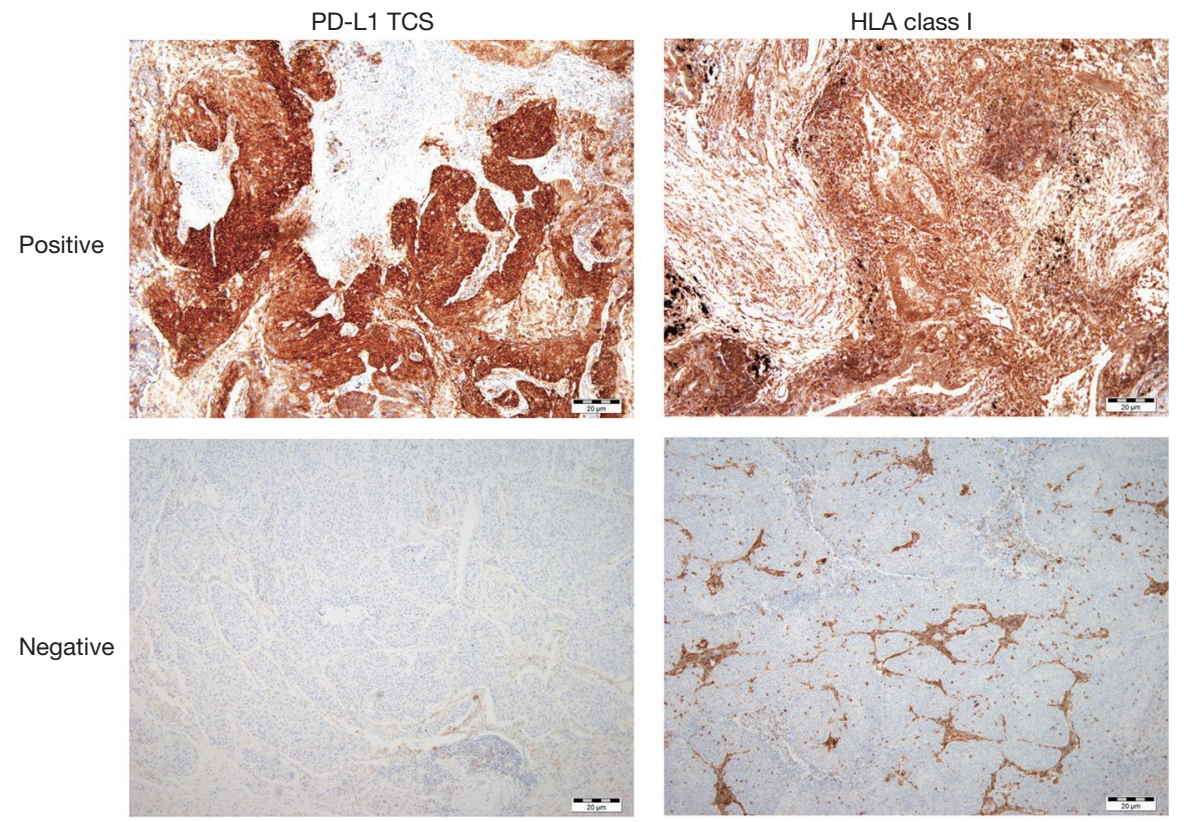

Figure 3 Representative positive and negative cases of the PD-L1 and HLA class I expression of cancer cells by using an immunohistochemistry staining are shown. PD-L1, programmed cell death protein ligand-1; TCS, tumor cells; HLA, human leukocyte antigen.
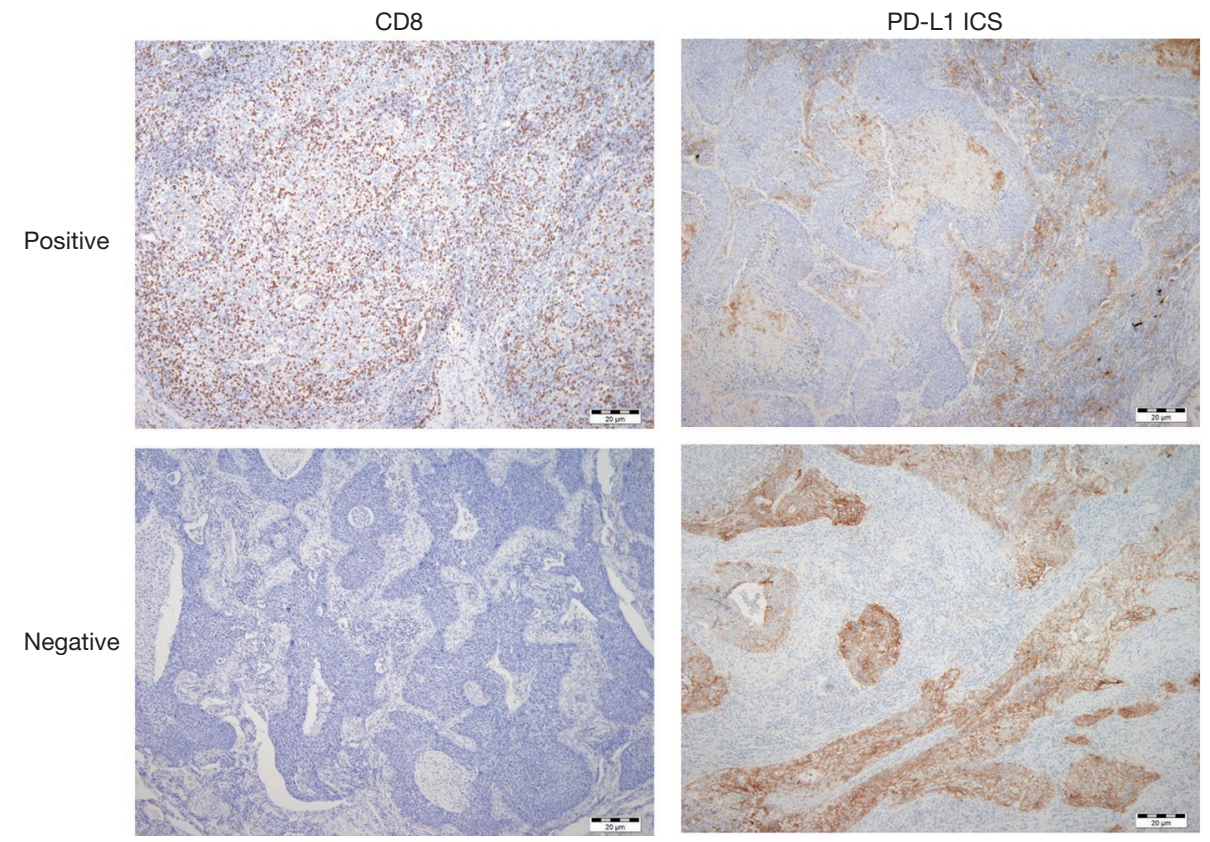

Figure 4 Representative positive and negative cases of the CD8 and PD-L1 of tumor infiltrating immune cells by using an immunohistochemistry staining are shown. PD-L1, programmed cell death protein ligand-1; ICS, immune cells. 

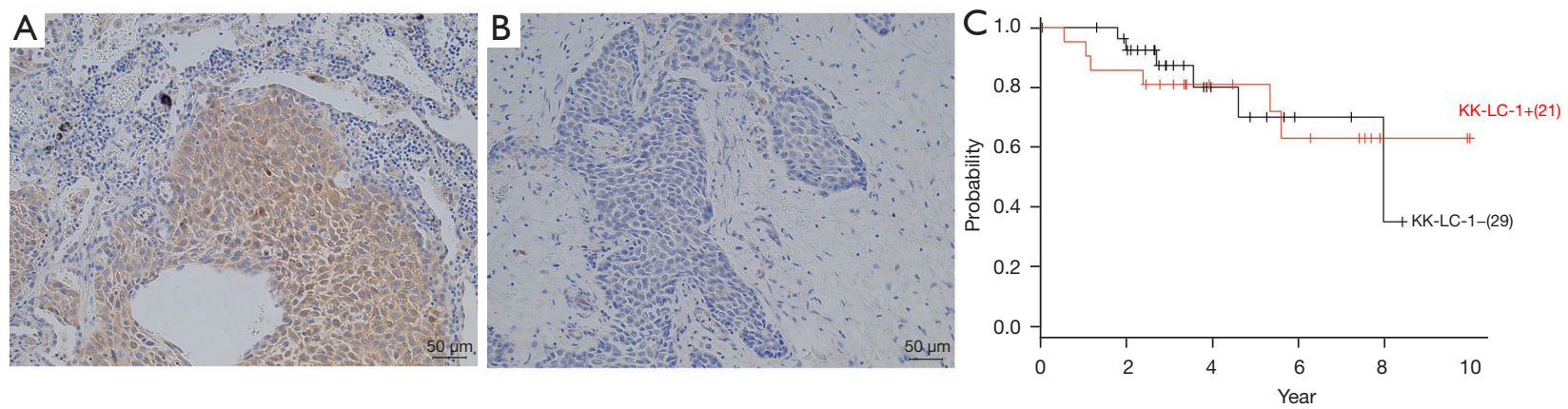

Figure 5 Analyses of the KK-LC-1 expression. (A) An immunohistochemistry staining image of KK-LC-1 in a case of KK-LC-1-positive lung squamous cell carcinoma. Nucleus and/or cytoplasm of lung squamous cell carcinoma cells are stained. (B) An immunohistochemistry staining image of KK-LC-1 in a case of KK-LC-1-negative lung squamous cell carcinoma. Neither the cell membrane nor the cytoplasm is stained. (C) Kaplan-Meier curves for the cancer-specific survival of lung squamous cell carcinoma patients with or without the KKLC-1 expression. There was no significant difference in prognosis depending on the intensity of KK-LC-1 expression in patients with lung squamous cell carcinoma. KK-LC-1, Kita-Kyushu Lung Cancer antigen-1.

\section{Discussion}

ICIs provide a survival advantage over conventional therapies for lung cancer treatment; however, more than $30-40 \%$ of patients receiving ICI monotherapy have progressive disease (PD) (4-8). We previously reported that 19 of 44 (43.2\%) non-small cell lung cancer (NSCLC) patients who received ICI monotherapy developed PD (12). It is also important to establish a new combined immunotherapy in order to further amplify the effects of ICI.

We have identified a shared cancer antigen, KK-LC-1, recognized by the cancer-specific CTL clone. KK-LC-1 is frequently expressed in many carcinomas (1). In the current analysis on the resected cases of lung squamous cell carcinoma, the expression of KK-LC-1 was observed in 21 of 50 cases (42\%), which corroborated our previous analysis. Furthermore, previous studies have reported positive expression of KK-LC-1 in 40 of 49 gastric cancers (81\%) (13) and 9 of 17 triple-negative breast cancer cases (53\%) (14). Additionally, dominant KK-LC-1specific immune response has also been observed in the cancer patients with complete regression after adoptive immunotherapy (3). KK-LC-1 is a powerful immunogenic antigen. We have measured various anti-KK-LC-1 polyclonal and monoclonal antibodies and have validated that Kmab34B3 is more precise and more sensitive to detect KK-LC-1 protein than other antibodies $(15,16)$.

However, the relationship between KK-LC-1 expression in cancer cells and patient prognosis remains unclear. In our previous investigation, the relationship between the expression of the cancer testis antigens, including MAGE-A3, MAGE-A4, NY-ESO-1 and KK-LC-1, and prognosis in 239 cases of NSCLC was analyzed. None of the cancer testis antigens have been found to significantly affect prognosis. However, lung cancer cases in which these four cancer antigens were not expressed at all exhibited a significantly poorer prognosis than those in which they were expressed (17). Jin et al. reported that there was no significant association between KK-LC-1 expression and patient prognosis after analysis of 38 resected lung cancer specimens (18). On the other hand, Chen et al. reported that high KK-LC-1 expression was an independent poor prognostic factor in hepatocellular carcinoma (HCC). KK-LC-1 regulated the Notch1/Hes 1 pathway and exacerbated HCC progression by physical interaction with presenilin-1 (19). In our analysis, the KK-LC-1 expression tended to be high in cancer cells with active cell proliferation on the basal side of the cancer tissue, suggesting a possibility of having a correlation between the proliferation ability of cancer cells and the KK-LC-1 expression in all four representative KK-LC-1 positive cases examined. Further analysis is needed to reach a conclusion.

We also observed that the prognosis might improve significantly if there was intratumoral infiltration of $\mathrm{CD}^{+}$ $\mathrm{T}$ cells in cases with KK-LC-1-positive lung squamous cell cancer. Our finding corroborated those of previous studies that reported a good prognosis in cancer cases in which $\mathrm{CD}^{+} \mathrm{T}$ cells infiltrated the tumor $(20,21)$. Naito et al. reported that various numbers of $\mathrm{T}$ cells with cytotoxic phenotype infiltrate in human colorectal cancer tissue, 


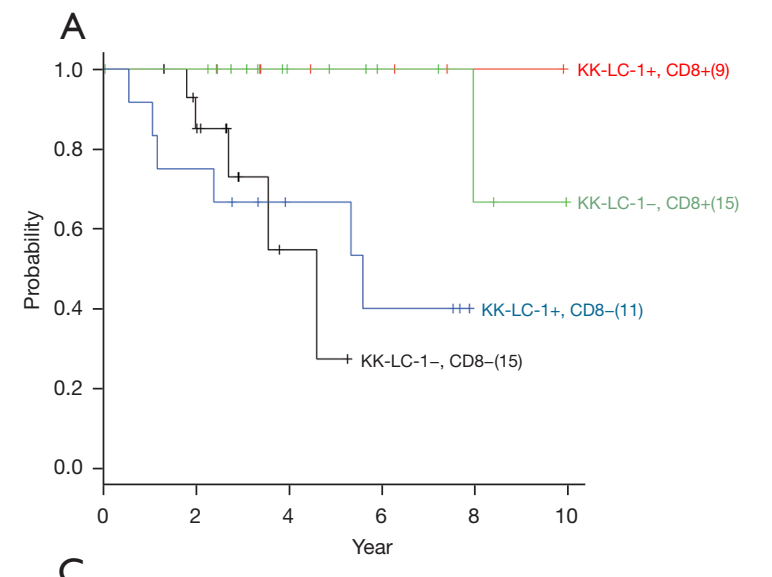

B
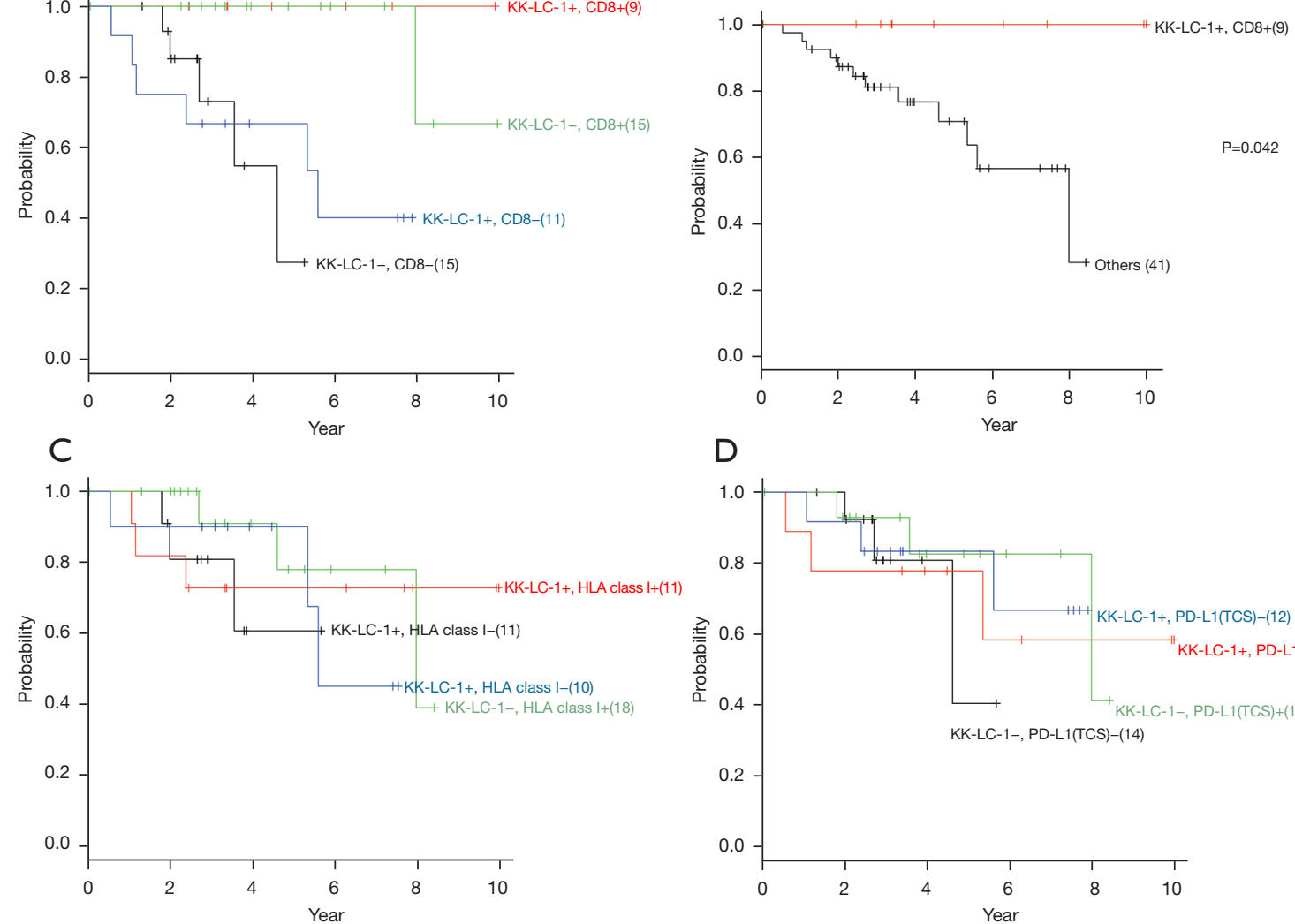

D

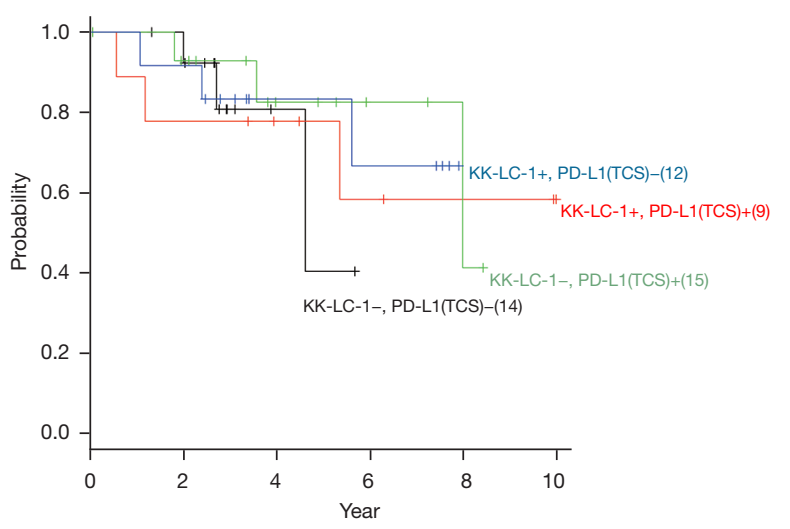

E

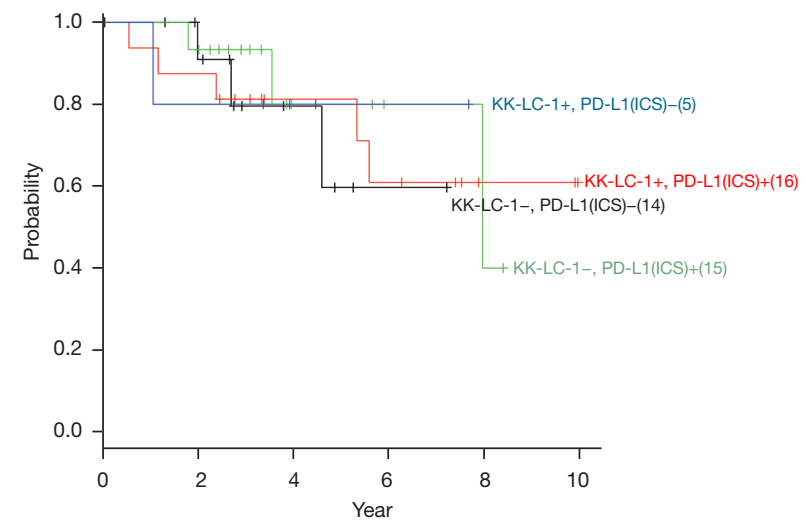

Figure 6 Survival curve with KK-LC-1 and each factor. (A) Kaplan-Meier curves for the cancer-specific survival of lung squamous cell carcinoma patients based on the KK-LC-1 expression and CD8 ${ }^{+}$T cell infiltration. No significant difference was observed. (B) Kaplan-Meier curves for the cancer-specific survival of lung squamous cell carcinoma patients with the KK-LC-1 expression and CD ${ }^{+} \mathrm{T}$ cell infiltration, and others. The cancer-specific survival of lung squamous cell carcinoma patients with the KK-LC-1 expression and CD $8^{+} \mathrm{T}$ cell infiltration was significantly better than others. (C) Kaplan-Meier curves for the cancer-specific survival of lung squamous cell carcinoma patients based on expression of KK-LC-1 and HLA class I. No significant difference was observed. (D) Kaplan-Meier curves for the cancer-specific survival of lung squamous cell carcinoma patients based on the expression of KK-LC-1 and PD-L1 in the cancer cells. No significant difference was observed. (E) Kaplan-Meier curves for the cancer-specific survival of lung squamous cell carcinoma patients based on the expression of KKLC-1 in cancer cells and PD-L1 in the tumor infiltrating immune cells. No significant difference was observed. KK-LC-1, Kita-Kyushu Lung Cancer antigen-1; HLA, Human leukocyte antigen; PD-L1, programmed cell death protein ligand-1; TCS, tumor cells; ICS, immune cells. 


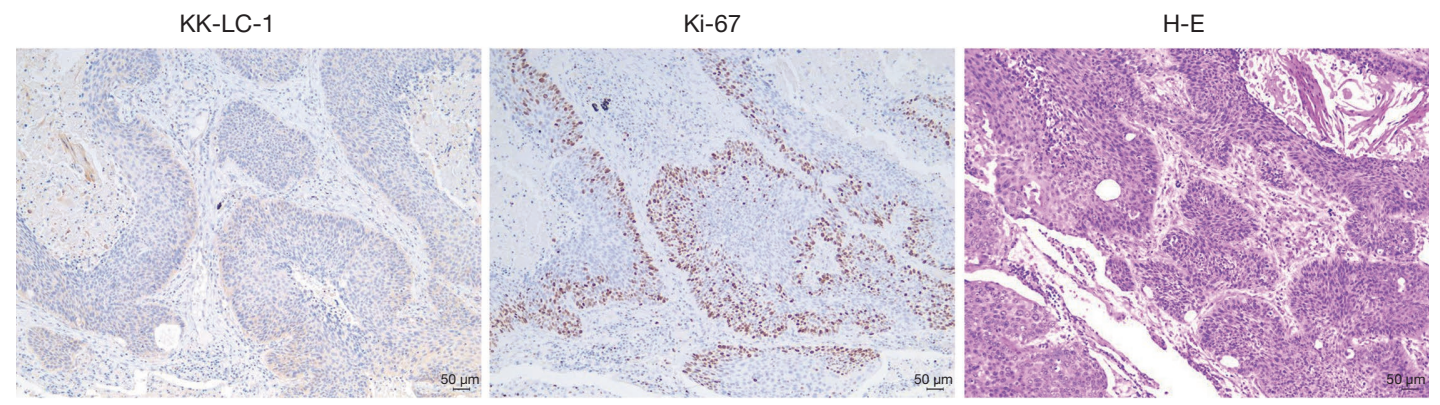

Figure 7 The left panel is KK-LC-1 and the center panel is Ki-67 stained during immunohistochemistry staining. The right panel shows HE staining. KK-LC-1, Kita-Kyushu Lung Cancer antigen-1; HE, Hematoxylin Eosin.

contributing to better patient survival (20). Among T lymphocytes, $\mathrm{CD}^{+} \mathrm{T}$ lymphocytes have a central effect of killing cancer cells and may be a sensitive biomarker. In a previous study (10), we found that the ratio of the area of $\mathrm{CD}^{+} \mathrm{T}$ lymphocytes to the area in the tumor stroma was more reflected in the prognosis, so we adopted this method in this study. This method is based on the literature (22).

We previously reported that mutated p 53 -specific CTLs and B cells infiltrated to tumor microenvironment and induced the cancer immune response (23). Functional analysis of tumor infiltrating $\mathrm{CD} 8^{+} \mathrm{T}$ cells is still required, since there is a possibility that the subset of $\mathrm{CD} 8^{+} \mathrm{T}$ cells may also include KK-LC-1-specific $\mathrm{CD}^{+} \mathrm{T}$ cells. In fact, we reported that HLA-B*1501- or 1507-restricted KK-LC1-specific CTL clone was present in the regional lymph nodes of lung cancer cases, killed KK-LC-1-positive lung cancer cells, and contributed to cellular cancer immune response (1). It has also been reported that HLA-*A0101restricted KK-LC-1-specific CTL clones are also present in cervical cancer patients (3). KK-LC-1 harbors multiple epitopes recognized by CTLs and is known to be a cancer antigen with high immunogenicity regardless of HLA type. We also reported that the transfer of the KK-LC-1-specific CTL-derived TCR into $\gamma \delta$ T cells acquires KK-LC-1specific cytotoxic activity. A marked T cell infiltration was observed in the tumors that exhibited a tumor regression effect (2). If there is no $\mathrm{CD}^{+} \mathrm{T}$ cell infiltration in KKLC-1-positive cancer cases, administration of KK-LC1-specific TCR-transferred $\gamma \delta$ T cells may elicit a cancer immune response. To the best of our knowledge, this is the first report showing $\mathrm{CD} 8^{+} \mathrm{T}$ cell infiltration as a potentially good prognostic factor in KK-LC-1-positive lung cancer.

The HLA class I molecule is an important molecule for antigen presentation to CTL. It was thought that cancer cells escape from the cell-mediated immune response via downregulation of the HLA class I molecule expression $(24,25)$. However, according to our previous report, the relationship between the HLA class I expression and prognosis in 403 NSCLC surgical cases was analyzed, and no significant difference was found between the intensity of expression and prognosis (26). In the present study, even if KK-LC-1 was expressed, the HLA-class I expression did not have any significant effect on patient prognosis.

Expression of PD-L1 in cancer cells suppresses the T cell immune function and induces the escape of cancer cells from the immune response (27). High expression of PD-L1 in tumor infiltrating immune cells also suggests an environment that suppresses the cancer immune response. The PD-1/PD-L1 signaling pathway was shown to transduce suppressive signals to $\mathrm{T}$ lymphocytes and promote immune evasion of cancer cells (28). We previously investigated the relationship between prognosis and the PD-L1 expression of tumor cells in 105 surgical patients with pulmonary neuroendocrine tumors. However, the PD-L1 expression status was not significantly correlated with the prognosis (29). Whether the PD-L1 expression in cancer cells affects prognosis remains controversial.

It has previously been reported that anti-CTLA-4 antibody enhances immune responses to NY-ESO-1, which is one the cancer testis antigens similar to KK-LC-1 $(30,31)$, and could induce NY-ESO-1-reactive $\mathrm{CD}^{+}{ }^{+} \mathrm{T}$ cells (32). In a phase I study involving a combination of NY-ESO-1 vaccine and anti-CTLA-4 Ab for treatment of NY-ESO1-positive melanoma, enhanced intratumoral $\mathrm{CD}^{+} \mathrm{T}$ cell proliferation was enhanced. In most cases, a cellular immune response against NY-ESO-1 was confirmed. It was reported that anti-CTLA-4 Ab might exhibit enhanced cell-mediated immune response against NY-ESO-1 (33). A more effective immunotherapy may be established by combining the cancer testis antigen-specific cancer immune response with ICI. 
This study has a few limitations. First, the sample size of the present study was small. Second, our study included some old specimens that were resected more than 10 years ago. However, it was confirmed that the cells of the old specimens were also stained by IHC. Although the possibility of poor staining with older specimens could not be ruled out, the adverse effects of older specimens were ignored. Larger cohort analysis is still required for further elucidation and validation of the results of this study.

In conclusion, our results indicated that a good prognosis might be expected in lung squamous cell cancer patients with the KK-LC-1-positive expression and the tumor infiltrating $\mathrm{CD}^{+} \mathrm{T}$ cells. There is a possibility that KKLC-1 expression might be highly expressed in cancer cells with high proliferative capacity. Further analysis is needed to conclude.

\section{Acknowledgments}

Funding: Dr. Ichiki reports grant support from JSPS KAKENHI (No. 18K08806 and 19K09294). Dr. Fukuyama reports grant support from Takeda Science Foundation.

\section{Footnote}

Reporting Checklist: The authors have completed the REMARK reporting checklist. Available at https://dx.doi. org/10.21037/tcr-21-1581

Data Sharing Statement: Available at https://dx.doi. org/10.21037/tcr-21-1581

Peer Review File: Available at https://dx.doi.org/10.21037/ tcr-21-1581

Conflicts of Interest: All authors have completed the ICMJE uniform disclosure form (available at https://dx.doi. org/10.21037/tcr-21-1581). The authors have no conflicts of interest to declare.

Ethical Statement: The authors are accountable for all aspects of the work in ensuring that questions related to the accuracy or integrity of any part of the work are appropriately investigated and resolved. This study was conducted in accordance with the Declaration of Helsinki (as revised in 2013). The study was approved by Saitama Hospital Ethics Committee (R2019-01) and informed consent was taken from all the patients.
Open Access Statement: This is an Open Access article distributed in accordance with the Creative Commons Attribution-NonCommercial-NoDerivs 4.0 International License (CC BY-NC-ND 4.0), which permits the noncommercial replication and distribution of the article with the strict proviso that no changes or edits are made and the original work is properly cited (including links to both the formal publication through the relevant DOI and the license). See: https://creativecommons.org/licenses/by-nc-nd/4.0/.

\section{References}

1. Fukuyama T, Hanagiri T, Takenoyama M, et al. Identification of a new cancer/germline gene, KK-LC-1, encoding an antigen recognized by autologous CTL induced on human lung adenocarcinoma. Cancer Res 2006;66:4922-8.

2. Ichiki Y, Shigematsu Y, Baba T, et al. Development of adoptive immunotherapy with KK-LC-1-specific TCRtransduced $\gamma \delta \mathrm{T}$ cells against lung cancer cells. Cancer Sci 2020;111:4021-30.

3. Stevanović S, Pasetto A, Helman SR, et al. Landscape of immunogenic tumor antigens in successful immunotherapy of virally induced epithelial cancer. Science 2017;356:200-5.

4. Brahmer J, Reckamp KL, Baas P, et al. Nivolumab versus Docetaxel in Advanced Squamous-Cell Non-Small-Cell Lung Cancer. N Engl J Med 2015;373:123-35.

5. Borghaei H, Paz-Ares L, Horn L, et al. Nivolumab versus Docetaxel in Advanced Nonsquamous Non-Small-Cell Lung Cancer. N Engl J Med 2015;373:1627-39.

6. Herbst RS, Baas P, Kim DW, et al. Pembrolizumab versus docetaxel for previously treated, PD-L1-positive, advanced non-small-cell lung cancer (KEYNOTE-010): a randomised controlled trial. Lancet 2016;387:1540-50.

7. Reck M, Rodríguez-Abreu D, Robinson AG, et al. Pembrolizumab versus Chemotherapy for PD-L1Positive Non-Small-Cell Lung Cancer. N Engl J Med 2016;375:1823-33.

8. Rittmeyer A, Barlesi F, Waterkamp D, et al. Atezolizumab versus docetaxel in patients with previously treated non-small-cell lung cancer (OAK): a phase 3, openlabel, multicentre randomised controlled trial. Lancet 2017;389:255-65.

9. Goldstraw P, Chansky K, Crowley J, et al. The IASLC Lung Cancer Staging Project: Proposals for Revision of the TNM Stage Groupings in the Forthcoming (Eighth) Edition of the TNM Classification for Lung Cancer. J Thorac Oncol 2016;11:39-51. 
10. Ichiki Y, Ueno M, Yanagi S, et al. An analysis of the immunological tumor microenvironment of primary tumors and regional lymph nodes in squamous cell lung cancer. Transl Lung Cancer Res 2021;10:3520-37.

11. Kanda Y. Investigation of the freely available easy-touse software 'EZR' for medical statistics. Bone Marrow Transplant 2013;48:452-8.

12. Ichiki Y, Taira A, Chikaishi Y, et al. Prognostic factors of advanced or postoperative recurrent non-small cell lung cancer targeted with immune check point inhibitors. J Thorac Dis 2019;11:1117-23.

13. Shida A, Futawatari N, Fukuyama T, et al. Frequent High Expression of Kita-Kyushu Lung Cancer Antigen-1 (KKLC-1) in Gastric Cancer. Anticancer Res 2015;35:3575-9.

14. Paret C, Simon P, Vormbrock K, et al. CXorf61 is a target for $\mathrm{T}$ cell based immunotherapy of triple-negative breast cancer. Oncotarget 2015;6:25356-67.

15. Takahashi Y, Fukuyama T, Futawatari N, et al. Expression of Kita-Kyushu Lung Cancer Antigen-1 as Detected by a Novel Monoclonal Antibody in Gastric Cancer. Anticancer Res 2019;39:6259-63.

16. Kondo Y, Fukuyama T, Yamamura R, et al. Detection of KK-LC-1 Protein, a Cancer/Testis Antigen, in Patients with Breast Cancer. Anticancer Res 2018;38:5923-8.

17. Shigematsu Y, Hanagiri T, Shiota H, et al. Clinical significance of cancer/testis antigens expression in patients with non-small cell lung cancer. Lung Cancer 2010;68:105-10.

18. Jin S, Cao S, Li J, et al. Cancer/testis antigens (CTAs) expression in resected lung cancer. Onco Targets Ther 2018;11:4491-9.

19. Chen Z, Zuo X, Pu L, et al. Hypomethylation-mediated activation of cancer/testis antigen KK-LC-1 facilitates hepatocellular carcinoma progression through activating the Notch1/Hes1 signalling. Cell Prolif 2019;52:e12581.

20. Naito Y, Saito K, Shiiba K, et al. CD8+ T cells infiltrated within cancer cell nests as a prognostic factor in human colorectal cancer. Cancer Res 1998;58:3491-4.

21. Chiba T, Ohtani H, Mizoi T, et al. Intraepithelial CD8+ T-cell-count becomes a prognostic factor after a longer follow-up period in human colorectal carcinoma: possible association with suppression of micrometastasis. Br J Cancer 2004;91:1711-7.

22. Rakaee M, Kilvaer TK, Dalen SM, et al. Evaluation of tumor-infiltrating lymphocytes using routine H\&E slides predicts patient survival in resected non-small cell lung cancer. Hum Pathol 2018;79:188-98.

23. Ichiki Y, Takenoyama M, Mizukami M, et al. Simultaneous cellular and humoral immune response against mutated p53 in a patient with lung cancer. J Immunol 2004;172:4844-50.

24. Cordon-Cardo C, Fuks Z, Drobnjak M, et al. Expression of HLA-A,B,C antigens on primary and metastatic tumor cell populations of human carcinomas. Cancer Res 1991;51:6372-80.

25. Campo MS, Graham SV, Cortese MS, et al. HPV-16 E5 down-regulates expression of surface HLA class I and reduces recognition by CD8 T cells. Virology 2010;407:137-42.

26. Hanagiri T, Shigematsu Y, Kuroda K, et al. Prognostic implications of human leukocyte antigen class I expression in patients who underwent surgical resection for nonsmall-cell lung cancer. J Surg Res 2013;181:e57-63.

27. Juneja VR, McGuire KA, Manguso RT, et al. PDL1 on tumor cells is sufficient for immune evasion in immunogenic tumors and inhibits CD8 T cell cytotoxicity. J Exp Med 2017;214:895-904.

28. Bour-Jordan H, Esensten JH, Martinez-Llordella M, et al. Intrinsic and extrinsic control of peripheral T-cell tolerance by costimulatory molecules of the CD28/B7 family. Immunol Rev 2011;241:180-205.

29. Ichiki Y, Matsumiya H, Mori M, et al. Predictive factors of postoperative survival among patients with pulmonary neuroendocrine tumor. J Thorac Dis 2018;10:6912-20.

30. Yuan J, Gnjatic S, Li H, et al. CTLA-4 blockade enhances polyfunctional NY-ESO-1 specific T cell responses in metastatic melanoma patients with clinical benefit. Proc Natl Acad Sci U S A 2008;105:20410-5.

31. Weber JS, Hamid O, Chasalow SD, et al. Ipilimumab increases activated $\mathrm{T}$ cells and enhances humoral immunity in patients with advanced melanoma. J Immunother 2012;35:89-97.

32. Kitano S, Tsuji T, Liu C, et al. Enhancement of tumorreactive cytotoxic $C D 4+T$ cell responses after ipilimumab treatment in four advanced melanoma patients. Cancer Immunol Res 2013;1:235-44.

33. Slingluff CL, Zarour HM, Tawbi HA, et al. A phase 1 study of NY-ESO-1 vaccine + anti-CTLA4 antibody Ipilimumab (IPI) in patients with unresectable or metastatic melanoma. Oncoimmunology 2021;10:1898105.

Cite this article as: : Ichiki Y, Fukuyama T, Ohmiya H, Ueno M, Yanagi S, Kanasaki Y, Goto H, Mikami S, Yamazaki H, Nakanishi K, Ishida T. Relationship between Kita-Kyushu Lung Cancer antigen-1 expression and prognosis of cases with lung squamous cell carcinoma. Transl Cancer Res 2021;10(12): 5212-5221. doi: 10.21037/tcr-21-1581 